Gut 1987, 28, S1, 265-268

\title{
Longterm pancreaticobiliary diversion stimulates hyperplastic and adenomatous nodules in the rat pancreas: a new model for spontaneous tumour formation
}

\author{
N H STACE*, T J PALMER, S VAJA, AND R H DOWLING \\ From the Gastroenterology Unit, Division of Medicine and Department of Pathology, UMDS of Guy's and \\ St Thomas' Hospitals, London
}

SUMMARY A model for spontaneous tumour formation in the rat pancreas is described that requires neither cocarcinogens nor dietary manipulation. Short term hypercholecystokininaemia, when induced by raw soya flour feeding, induces benign and malignant tumours of the rat pancreas. Pancreaticobiliary diversion (PBD) results in hypercholecystokininaemia and in the short term, pancreatic hyperplasia. Longterm PBD was done to establish whether hypercholecystokininaemia thus produced would also lead to pancreatic neoplasia. After a period of 16-21 months hyperplastic and adenomatous nodules, one of the latter showing carcinoma in situ, were found in PBD rats but not in sham operated control rats.

Short term (1-8 weeks) pancreaticobiliary diversion (PBD) in the rat stimulates pancreatic hyperplasia ${ }^{1}$ and exocrine secretory hyperfunction, ${ }^{2}$ probably as a result of secondary hypercholecystokininaemia. ${ }^{3}$ Short term raw soya flour (RSF) feeding also stimulates pancreatic hyperplasia and leads to an increase in plasma immunoreactive cholecystokinin (CCK) concentrations. ${ }^{4}$ In long term studies, RSF feeding induces benign and malignant tumours in the rat pancreas. ${ }^{5}$ We wished to see, therefore, if longterm PBD would induce similar pancreatic tumours in the rat.

\section{Methods}

\section{ANIMALS}

Male and female SPF Wistar rats, weighing approximately $250 \mathrm{~g}$ were used. Pancreaticobiliary diversion was achieved by interposing $50 \mathrm{~cm}$ of jejunum, isoperistaltically, between the pylorus and the ampulla of Vater. ${ }^{1}$ Controls (TrC) were established by transecting and reanastomosing the intestine as for PBD, but with preservation of the normal intestinal anatomy. The animals were housed under conventional laboratory conditions and fed a standard rat chow (No. 1, Beta Diets, Essex).

Address for correspondence: Professor R H Dowling, Gastroenterological Unit, 18th Floor, Guy's Tower, Guy's Hospital, London SE1 9RT.

* Present address: Dept. of Med., Clinical School of Medicine, Wellington Hospital, Wellington, New Zealand.
Sixteen to 21 months after surgery, the rats were killed by cardiac aspiration under pentobarbitone anaesthesia. The pancreas was dissected out, dabbed dry and weighed. The presence of macroscopic nodules was noted and portions of the head, body and tail of the pancreas were fixed in formalin. After routine processing, $5 \mu \mathrm{m}$ sections were cut at three levels and stained with haematoxylin and eosin, by van Gieson's method for collagen and by Adams' DMAB-nitrate method for tryptophan (to show zymogen granules). The presence or absence of nodules was recorded and the distinction between hyperplastic and adenomatous nodules made according to Levison et al. ${ }^{6}$ The distribution of zymogen granules, the number of mitoses and the degree of cellular pleomorphism were assessed, and other abnormalities recorded.

\section{Results}

\section{MACROSCOPIC FINDINGS}

Body and pancreatic weights are shown in the Table. The mean pancreatic wet weight in the pancreaticobiliary diversion (PBD) rats was $108 \%$ greater than that in the $\operatorname{TrC}$ rats $(\mathrm{p}<0.01)$. This difference was more striking when pancreatic wet weight was expressed as mg per $100 \mathrm{~g}$ body weight $(134 \%$; $\mathrm{p}<0.001)$. Macroscopic nodules were seen in six of the PBD rats but in none of the TrC animals. The 
Table Body and pancreatic weights (means $\pm S E M$ ) in transected controls $(\operatorname{TrC})$ and in rats with pancreaticobiliary diversion (PBD) studied 16-21 months postsurgery.

\begin{tabular}{lccc}
\hline & $T R C$ & $P B D$ & $P$ \\
\hline $\mathrm{n}$ & 12 & 13 & \\
$\begin{array}{l}\text { Body weight (g) } \\
\begin{array}{c}\text { Pancreatic wet } \\
\text { weight (mg) }\end{array}\end{array}$ & $494 \pm 23$ & $448 \pm 19$ & $N S$ \\
$\begin{array}{c}\text { Pancreatic weight } \\
\text { (mg/100 g body weight) }\end{array}$ & $243 \pm 9$ & $570 \pm 83$ & $<0.001$ \\
\hline
\end{tabular}

largest pancreas weighed $6094 \mathrm{mg}$ and contained three nodules, with a maximum diameter of $7 \mathrm{~mm}$ (Fig. 1).

\section{MICROSCOPIC FINDINGS}

\section{Transected controls}

Upon microscopy, no nodules were found in the 15 transected controls. In six, however, there was focal lobular atrophy and mild inflammation; evidence of vasculitis was seen in one rat. These appearances are in keeping with ageing. No mitoses were seen and the distribution of zymogen granules was normal.

\section{$P B D$}

In the PBD rats, nodules were seen in 11 of the 15 pancreata. All 11 contained hyperplastic nodules and four of the 11 contained adenomatous nodules. One adenomatous nodule showed extension of fibrous tissue into the nodule, breaking it up into smaller groups of acini; at no point was the capsule invaded. The nodules measured up to $7 \mathrm{~mm}$ in diameter.

The mitotic activity was variable with up to 20 mitoses per 10 high power fields (HPF) in the adenoma containing the fibrous stroma. Five mitoses in one HPF were present in another nodule. Mitoses were present in hyperplastic and adenomatous nodules and atypical forms were seen in both. A degree of nuclear pleomorphism was invariably present in the nodules but was greater in the adenomata. There was no consistent change in the distribution or the quantity of zymogen granules within the acinar cells. No

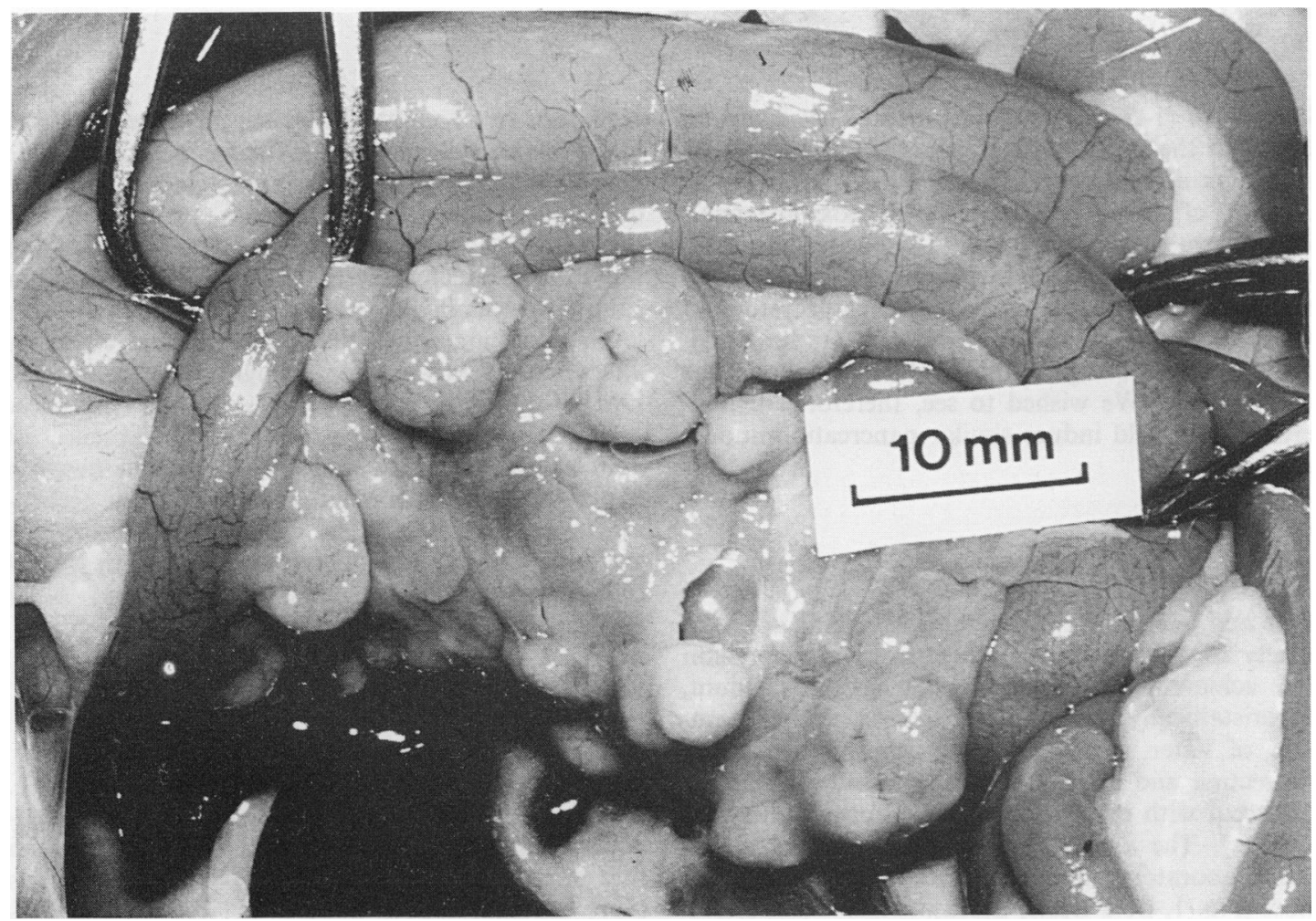

Fig. 1 Underside of duodenal loop (retracted by steel forceps) displaying the pancreas studded with macroscopic nodules in a rat with $P B D$. 


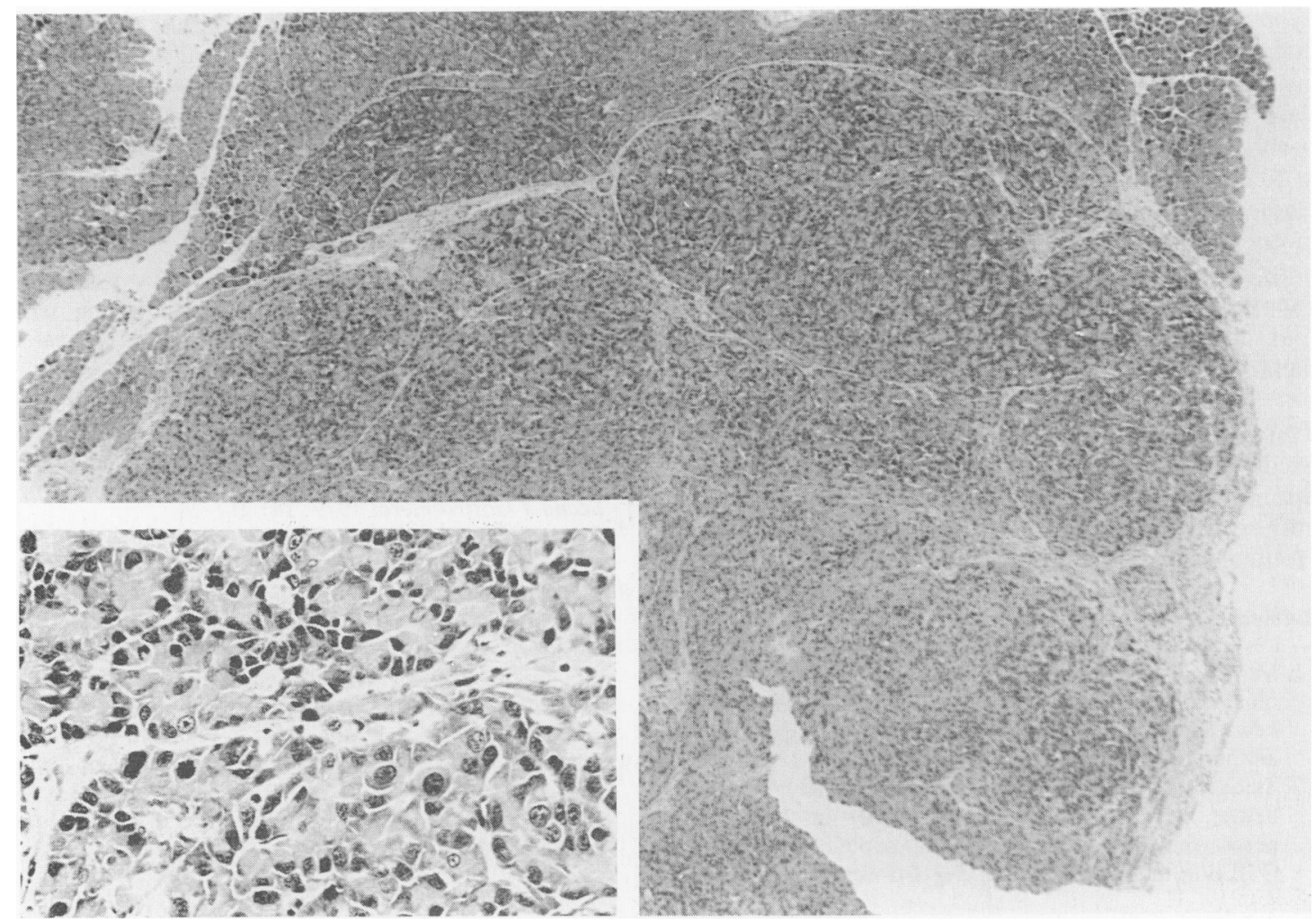

Fig. 2 Adenomatous nodule from PBD rat, showing fibrous capsule and extension of fibrous stroma into nodule $(H+E \times 40)$. Inset $(H+E \times 50)$ shows striking nuclear pleomorphism, mitoses and disordered acinar architecture.

invasive carcinoma was seen, although the nodule with the fibrous stroma was considered to show carcinoma in situ (Fig. 2).

The distribution of nodules appeared random in the gland and a maximum of 11 nodules was seen in a single pancreas. The presence of adenomata was not necessarily associated with large numbers of hyperplastic nodules. Signs of ageing were seen in two PBD rats.

\section{Discussion}

These data extend our previous short term PBD studies and show that after this procedure, the rat pancreas not only becomes hyperplastic but also shows nodule formation. In addition to the hyperplasia, the majority of the PBD rats developed hyperplastic nodules and in some there were also adenomatous nodules. It is of note that spontaneous tumour formation is exceedingly rare in the rat pancreas. ${ }^{7}$ Indeed, although nodular hyperplasia has occasionally been observed in older rats, this was not found in the transected controls of the present study nor in the control rats in the soya flour study. ${ }^{4}$

Although the number of animals in the present study was small and groups were not studied systematically at different times after PBD, the present observations are similar to those made when raw soya flour was fed over the long term. ${ }^{5}$ Both PBD and RSF feeding are associated with raised plasma concentrations of immunoreactive CCK.$^{3,4}$ Nodules, progressing to invasive carcinoma, have also been described after a prolonged course of CCK injections. ${ }^{6}$ Further studies are needed to document the chronology of nodule formation, for comparison with observations already made in RSF-fed rats, ${ }^{5}$ and to relate the changes observed to parameters of normal pancreatic function.

No invasive tumours were found in this study but the duration of PBD (16-21 months) was less than the $90+$ weeks required for the production of invasive carcinomas in response to a RSF diet. ${ }^{5}$

The present study, and the work presented by Miazza et al. in these proceedings, ${ }^{8}$ suggest that 
hypercholecystokininaemia alone is capable of inducing neoplastic changes without the need for an initiator such as azaserine. McGuiness and Wormsley ${ }^{9}$ have also presented evidence to support this conclusion, as CCK produced an irreversible change in azaserine-induced foci after 12 weeks RSF diet. Cholecystokinin is thus both an initiator and promotor of pancreatic tumours.

In conclusion, we have described a new model for spontaneous tumour formation in the rat pancreas that does not require the use of cocarcinogens or dietary manipulation.

This work was supported, in part, by grants from the Medical Research Council of New Zealand and the Special Trustees of Guy's Hospital to whom the authors are grateful. Thanks are also due to Mrs Ann Hollington for secretarial help.

\section{References}

1 Miazza B, Levan H, Vaja S, Dowling RH. Pancreaticobiliary diversion (PBD) by duodenal transposition: a new model for stimulating jejunal and pancreatic adaptation. [Abstract] Gut 1980; 21 : A917.

2 Stace NH, Vaja S, Murphy GM, Dowling RH. Bigger is better! Secretory dose-response studies in the hyperplastic pancreas following pancreaticobiliary diversion (PBD) in the rat. [Abstract] Dig Dis Sci 1983; 28 : 949.
3 Miazza B, Levan H, Ghatei M, Adrian T, Bloom S, Dowling RH. Role of regulatory peptides in the small bowel and pancreatic adaptation of pancreaticobiliary diversion (PBD) following jejunal or ileal transposition in the rat. [Abstract]. Eur J Clin Invest 1982; 12: 27.

4 Adrian TE, Pasquali C, Pescosta F, Bacarese-Hamilton AT, Bloom SR. Soya-induced pancreatic hypertrophy and rise of circulating cholecystokinin. [Abstract] Gut 1982; 23: A889.

5 McGuiness EE, Morgan RGH, Levison DA, Frape DL, Hopwood D, Wormsley KG. Effects of long term feeding of soya flour on the rat pancreas. Scand $J$ Gastroenterol 1980; 15: 497-502.

6 Levison DA, Morgan RGH, Brimacombe JS, Hopwood D, Coghill G, Wormsley KG. Carcinogenic effects of di (2-hydroxypropyl) nitrosamine (DHPH) in male Wistar rats; promotion of pancreatic cancer by soya flour diet. Scand J Gastroenterol 1979; 14: 217-24.

7 Altman NH, Goodman DG. In: The laboratory rat. New York: Academic Press, Inc, 1979: 342-76.

8 Miazza BM, Lindgren S, Chayvialle JA, Nicholet T, Loizeau E. Appearance of nodular lesions in the exocrine pancreas following pancreaticobiliary diversion in the rat: an effect of elevated cholecystokinin plasma levels. [Abstract 75]. 3rd International Conference of Intestinal and Pancreatic Adaptation, Titisee 1986. (In Press)

9 McGuiness EE, Wormsley KG. Regression of pancreatic nodules. [Abstract 77]. 3rd International Conference on Intestinal and Pancreatic Adaptation, Titisee 1986. [In Press] 